\title{
Into, Through, and Beyond: An Integrative Framework for the Teaching of Statics
}

\author{
Jon-Michael Hardin \\ Virginia Military Institute
}

\section{Introduction}

Statics is universally recognized as a fundamental foundation course in engineering. Therefore, much research has focused on specific pieces of the Statics teaching puzzle, such as Statics Concept Inventories, concept-based hands-on experiments or demonstrations, and engineering design. However, there has been little discussion concerning a pedagogical framework by which to integrate those pieces to achieve the primary goal of overall student competency in Statics. In this paper, the author puts forth such a framework: Into, Through, and Beyond, a pedagogically-based teaching methodology that has been used primarily in the teaching of reading, ESL (English as a Second Language), and, more recently, in the teaching of foreign languages. Additionally, through its current linguistic usage, the content-based Into, Through, and Beyond framework provides an effective, analogous, and new model for the teaching of the Statics engineering 'language,' composed of specific vocabulary (e.g., force, moment, couple) and grammar (e.g., the 'rules' for constructing a free-body diagram and mathematic rules), within the content-base of fundamental engineering concepts (e.g., equivalency and equilibrium) and engineering design.

In this paper, the author discusses the Into, Through, and Beyond teaching methodology and provides a specific example for the use of this methodology in the teaching of Statics. It should be noted, however, that the provided example should not be viewed as a specific model since it has not been formally tested in classroom usage. Rather, this example is presented solely to illustrate how the Into, Through, and Beyond methodology could be adapted for use in the teaching of Statics.

\section{Into, Through, and Beyond}

The Into, Through, and Beyond teaching method evolved from research that supported the effectiveness of content-based instruction (CBI) in the teaching of reading. This research showed that student learning and retention of linguistic skills, i.e., the effective use of vocabulary and grammar, was improved when these linguistic basics were taught within a content-based framework. Student learning and retention of the content materials was similarly shown to be improved through use of this teaching method. Based on the success of this method in the teaching of reading, the method was later adapted to the teaching of ESL and of foreign languages. ${ }^{1,2}$

The Into, Through, and Beyond teaching framework is based on the iterative use of a progressive three-stage 'scaffolded' cycle with each stage designed to motivate and 
ensure student progress in the comprehension and mastery of the specific content and requisite linguistic skills presented in each cycle. Student self-learning through discovery, either individually or in groups, is an essential element of each of the three stages of the cycle. Within its current use in linguistics, the three stages of a cycle are:

1. Into: The goal of this stage is "for students to gain an entrée into the topic, recognize the depth of their own prior knowledge, and be better prepared for the new content materials they are about to encounter."3 By bringing their own knowledge of the content topic forward, students are not only better prepared for the new content, but have an investment in the further learning of that content. Additionally, by probing the student's prior knowledge of the content area, the instructor can better tailor the content lessons to the specific student group and have a greater understanding of the student misconceptions that must be overcome through the content area instruction. Typical Into activities include "reviews of previously learned content, the use of content-related visuals, reaction journals, vocabulary previews, free association or visualization exercises, and anticipation reaction guides to assist students in accessing the new content material.",

2. Through: The goal of this stage is for students to practice new skills and to demonstrate comprehension and competency in the topic content area. In this stage, "students encounter the new material, relating it to their discussions during the Into stage. This may entail confirming or rejecting the hypotheses they formed or expanding their knowledge base..." Relating new content material to the Into discussions provides a 'scaffold' by which students can better learn and retain new vocabulary, grammar, and more fully comprehend new concepts. Typical Through activities include "grammar development or vocabulary expansion, reading guides..., and information gap tasks..."6

3. Beyond: The goal of this stage is for students to "demonstrate both conceptual and linguistic mastery"7 of the content material by creatively applying their new knowledge of that material. This stage is essential to the learning and retention of new content materials since it provides the 'scaffolding' step that motivates students to see the use of the content materials beyond the mere learning of those materials to their relevance in a variety of contexts. Typical Beyond activities are based on more extended and varied uses of the newly learned content materials.

Although use of the Into, Through, and Beyond teaching method is currently limited to linguistic-based instruction, the essential 'scaffolded' structure of the method provides a framework that can be effectively adapted to the teaching of Statics. 


\section{Into, Through, and Beyond for the Teaching of Statics}

The Into, Through, and Beyond method is, at its core, a content-based instruction methodology. However, the method is independent of any specific content. This content independence is what has allowed the method to be successfully adapted from its original use in the teaching of reading to the teaching of ESL and foreign languages. Similarly, the method can be adapted successfully to the teaching of Statics.

The linguistic use of the method, however, does provide a necessary model for the distinctive separation of 'linguistic skills' (e.g., spelling, vocabulary and grammar) and 'conceptual content' that may be quite effective in the organization of Statics instruction. For example, much work has been done in the development of Statics Concept Inventories. In defining the essential 'conceptual content' knowledge base required for the mastery of Statics, these concept inventories essentially have limited the definition of the 'skills' required for Statics as mathematic skills. However, it may be more appropriate in the teaching of Statics to define 'skills' and 'concepts' in a different way, more in line with an analogous linguistic form, than currently defined in the Statics Inventory Concepts methods, for example:

\section{Skills:}

Vocabulary: Forces, Moments, Couples, Vectors, etc...

Grammar/Rules: Free-body diagram 'rules', Mathematic 'rules' (vector components, vector cross-product, etc...), Mechanics 'rules' (forces equal in magnitude and opposite in direction, the differential relationships between load, shear and moment, etc...)

Concepts: System equivalency, Equilibrium, Engineering design

This redefining of Statics 'skills' and 'concepts' is based on what has been found to be the most effective feature of content-based instruction methodologies: that students are more motivated and, therefore, better learn and retain both 'skills' and 'concepts' when the skills are taught within the broader context of concept content material.

It is not surprising that students are more motivated to learn a 'skill' when they can clearly and simultaneously see, within the context of the 'concept content,' why they need to know the skill and how that skill may be used within the broader 'concept content.' So, for example, students may be more motivated to learn and retain knowledge about the physical nature of forces and how to mathematically express their vector components, if those 'skills' are taught within the broader context of the 'concept content' of equilibrium or engineering design. If not taught within a broader 'concept' context, the learning of those skills becomes mere 'hoop jumping,' a syndrome known to many engineering instructors by which students, for example, can write a force vector but have no idea of why or when or actually how to use such knowledge in the formulation 
and solution of an engineering problem. While hands-on demonstrations or exercises may aid in student understanding of the physical nature of forces, for example, the link between that understanding and the formulation and solution of an engineering problem can remain tenuous, at best. By using the definitions of Statics 'skills' and 'concepts' given above, the content-based Into, Through, and Beyond instruction method may provide students, as it has in its linguistic usage, the motivation to learn and retain both the 'skills' and the 'concepts' required to comprehend and master Statics.

\section{Statics Lesson-Planning Example Using Into, Through and Beyond}

In addition to its clear distinction between 'skills' and 'concept content', the Into, Through, and Beyond method makes use of a 'scaffolded' approach to student learning, whereby prior knowledge supports the acquisition of new skills and concept comprehension, which in turn are expanded and deepened through usage beyond their acquisition. Each cycle then is rigorously structured such that it provides the 'prior knowledge' support for the next cycle.

In this section, an example showing several iterations of the Into, Through, and Beyond method is presented to show how this method may be used for the instruction of Statics. As noted earlier, this example should not be viewed as a specific model for instruction, since it has not been formally tested in actual classroom usage, but instead should be considered merely as adding some, although certainly not all, 'engineering meat' to the skeletal framework of the method to show a possible configuration for its use in Statics instruction. Additionally, it should be noted that the class periods required for any specific iteration may vary from less than one class period to several class periods.

The first iterations of the Into, Through, and Beyond method in Statics instruction should present the concept of engineering design, the broadest 'concept content' context for the learning of Statics skills and concepts. Within this concept context, students gain an understanding of why they need to gain knowledge of Statics skills and concepts.

1. Concept content: Engineering Design, Force Equilibrium

Vocabulary: forces, equilibrium, Statics

Grammar: requirements for general force equilibrium (i.e., forces must 'balance' one another)

\section{Into Activities:}

Discussion of general engineering design constraints (group/class)

Discussion of static equilibrium in engineering design/Statics (class)

Discussion of forces within context of equilibrium (class)

Examples of force equilibrium in engineering design case studies

\section{Through Activities:}


Hands-on demo of forces in 2-D space (can include forces that are equal in magnitude and opposite in direction with minimal discussion) (student/group)

Hands-on demo of equilibrium of forces in 2-D space (student/group)

\section{Beyond Activities:}

These activities should be designed to encourage students to use their new knowledge of the nature of forces within the broader context concepts of the balancing requirements of force equilibrium and of engineering design:

Paper solution for a simple force equilibrium design problem in 2-D space (group project)

Class discussion of each group's paper design

Physical fabrication of paper design (group project/group discussion)

Class discussion of each group's physical solution

2. Concept Content: Engineering Design, Force and Moment Equilibrium Vocabulary: moment, particle, rigid body

Grammar: particle/force equilibrium requirement (forces must balance one another), rigid body/force and moment equilibrium requirements (forces must balance one another and moments must balance one another)

\section{Into Activities:}

Discussion of prior design solutions (from iteration 1) with respect to moment equilibrium and difference between particle and rigid body equilibrium requirements (group/class)

Discussion of static rigid body equilibrium in engineering design/Statics (class)

Examples of force and moment equilibrium in engineering design case studies

\section{Through Activities:}

Hands-on demo of moments (can include moments and couples) (student/group)

Hands-on demo of force and moment equilibrium/particle vs. rigid body equilibrium requirements (student/group)

\section{Beyond Activities:}

These activities should encourage students to use their new knowledge of moments and the distinction between particles and rigid bodies within the context concepts of rigid body equilibrium balancing requirements and of engineering design:

Paper solution for a simple 2-D rigid body equilibrium design problem (group project) 
Class discussion of each group's paper design

Physical fabrication of paper design (group project/group discussion)

Class discussion of each group's physical solution

The following iteration focuses on the use of engineering/mathematical skills and engineering concepts/principles in the engineering formulation and solution of physical problems. It is essential at this stage for students to be informed explicitly that 1) engineering and mathematical formulation and solution are what separates engineering from empirical problem solving (i.e., this is what engineers actually do as 'engineers') and 2) the engineering concepts and the engineering/mathematical skills being learned in the Statics course are necessary to be able to perform engineering design and solution beyond the course. Since Statics is one of the first engineering courses in most engineering curricula, student comprehension of engineering problem formation and solution, and the requisite accompanying skills and concepts, as the foundation of engineering design, is best gained at this early stage of the student's academic training so that this core concept and motivation may be carried over by the student into later engineering courses.

3. Concept Content: Engineering vs. empirical solution for Engineering Design Vocabulary: Free-body diagram (FBD), vector, engineering principles

Grammar: flow chart of engineering formulation and solution

\section{Into Activities:}

Discussion of prior design solutions (from iteration 1 and 2) with respect to empirical solution methods (class)

Discussion of engineering versus empirical solution including vocabulary preview of FBD, vectors, and engineering principles (class)

\section{Through Activities:}

Fill-in flow chart of empirical versus engineering formulation and solution (see Figure 1 Flow Chart: Empirical versus Engineering Formulation and Solution, below) (Note: This activity should explicitly highlight the differences between engineering formulation and solution versus empirical solution, as discussed above)

Present and briefly discuss examples of FBDs and vector representation of forces

\section{Beyond Activities:}

These activities should be designed to highlight the difficulties of empirically solving a complex engineering problem and thus serve to provide motivation for student learning of the two skills, drawing FBD and vector representation of forces, that they will encounter in the following iterations.

Paper solution for a more complex 2-D rigid body equilibrium design problem (group project) 
Class discussion of each group's paper design

Physical fabrication of paper design (group project/group discussion)

Class discussion of each group's physical solution

Class discussion of engineering versus empirical formulation and

solution using engineering design case study examples.

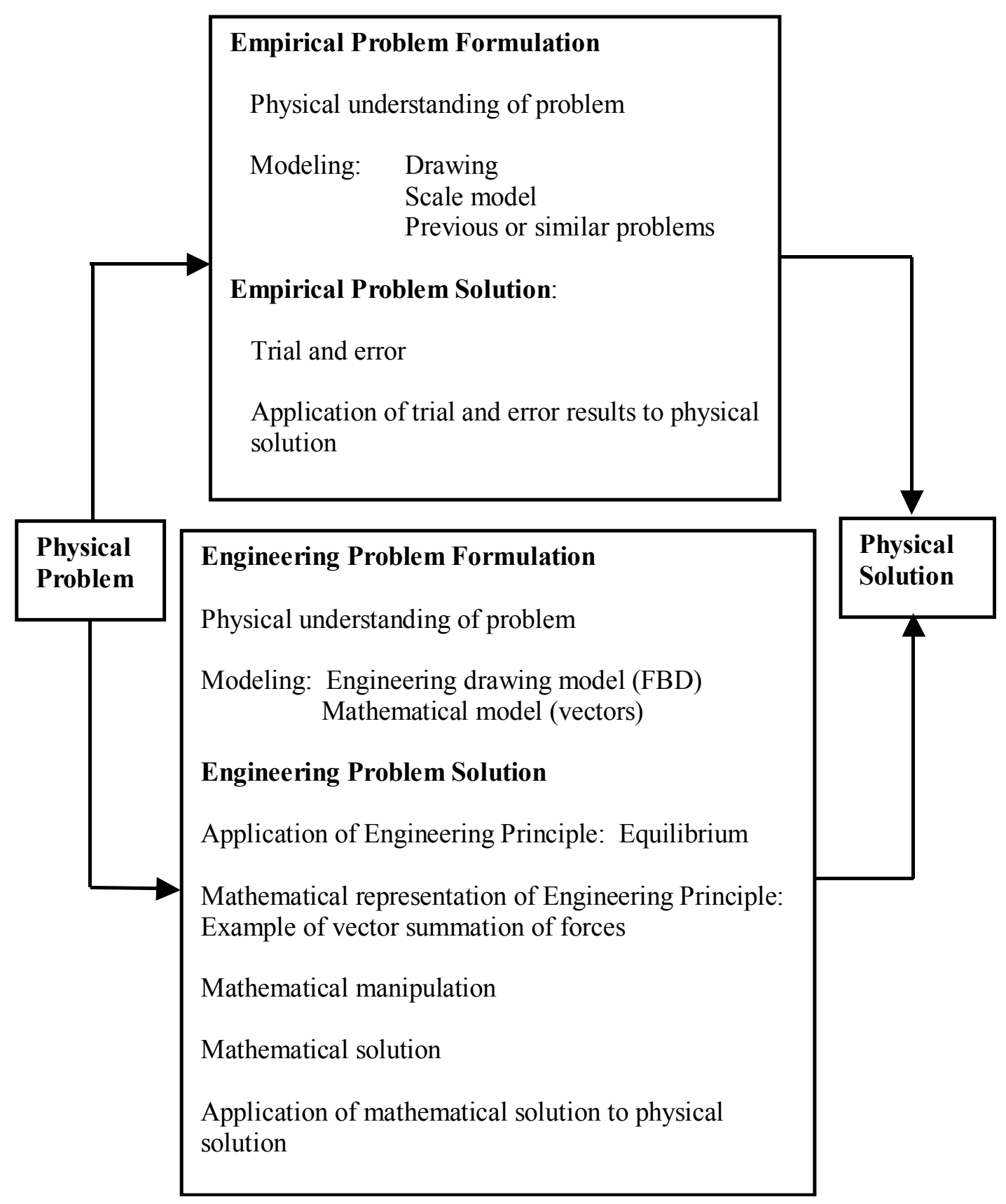

Figure 1 Flow Chart: Empirical versus Engineering Formulation and Solution (see Iteration 3/Through Activities, above) 
The following iteration focuses on the use of the free-body diagram in the formulation and solution of engineering problems.

4. Concept Content: Engineering formulation/solution for Engineering Design

Vocabulary: Free-body diagram (FBD)

Grammar: FBD 'rules'

\section{Into Activities:}

Discussion of FBD with examples (class)

Discuss possible 'rules' for drawing FBD, based on examples

(group/class)

\section{Through Activities:}

Discuss actual FBD 'rules' (class)

Practice drawing FBD of physical problems (group)

Discuss practice FBD drawings made (class)

Discussion of prior design formulations and solutions (from iterations 2 and 3) with respect to FBD (group/class)

\section{Beyond Activities:}

These activities should be designed to provide students the opportunity to practice their new knowledge of drawing an FBD within an engineering design context.

Paper formulation and solution, using FBD, for a more complex 2-D rigid body equilibrium design problem (group project)

Class discussion of each group's paper design with emphasis on FBD Physical fabrication of paper design (group project/group discussion)

Class discussion of each group's physical solution

The following iterations focus specifically on the skills associated with the mathematical expression of forces and equilibrium requirements and highlight the relation of those mathematical expressions to particle/force equilibrium solutions in engineering design.

5. Concept Content: Force Equilibrium

Vocabulary: forces, vectors,

Grammar: vector representation of forces, mathematical expression for force equilibrium

\section{Into Activities:}

These activities should highlight that the single skill of drawing an FBD, although necessary, is not sufficient to solve complex engineering problems and, therefore, additional skills must be learned.

Paper problem formulation, using FBD, for a more complex 2-D problem (group) 
Class discussion of paper problem formulations.

Discussion of paper problem solution (group/class)

Class discussion of vectors (vocabulary preview)

\section{Through Activities:}

Discussion of vectors as a mathematical representation of forces (class)

Practice writing force vectors in the form $\vec{F}=F \nabla_{\theta}$ (group)

Discussion of mathematical expression of force equilibrium

requirements $(\Sigma \vec{F}=\overrightarrow{0})$

\section{Beyond Activities:}

These activities should be designed to provide students the opportunity to practice their prior knowledge of drawing FBD and their new knowledge of writing force vectors within an engineering design context.

Paper formulation, using FBD and force vector expression, for a more complex 2-D rigid body equilibrium design problem (group project)

Class discussion of paper problem formulations

6. Concept Content: Force Equilibrium, Force Equivalency

Vocabulary: forces, vectors, vector addition, force/vector components

Grammar: vector addition rules (parallelogram law and triangle rule), rules for resolving force/vector into components, force equivalency rules

\section{Into Activities:}

These activities should explicitly highlight that the representation of forces as vectors and the force addition necessary to ensure force equilibrium requires that students learn such mathematical skills as a) how vectors are added and $b$ ) how to write force vectors in their rectangular component form to facilitate vector addition.

Students may become confused in their study of Statics if they can not see any connection between the various activities associated with the mathematical representation and manipulation of vectors, and the physical formulation and solution of an engineering problem. Care should be taken to ensure that such a connection is clearly and explicitly provided for the students at this stage so as not to create the initial environment in Statics that leads to student 'hoop jumping.'

Discussion of paper problem solution, based on FBD, vector representation of forces, and mathematical expression of force equilibrium requirements (group/class)

Class discussion of vector addition (grammar preview)

Class discussion of force system equivalency (concept preview) 


\section{Through Activities:}

Hands-on demos and discussion of vector addition (group)

Hands-on demo of force component equivalency of the relationship

$$
F \angle \theta=F_{X} \longrightarrow+F_{Y} \uparrow \quad \text { (group) }
$$

Discuss expressing force vectors in the component form

$$
\vec{F}=F_{X} \longrightarrow+F_{Y}^{\uparrow} \text { (group) }
$$

Hands-on demo of using component form of force vector for equilibrium requirements $\left(\sum F_{X}=0\right.$ and $\left.\Sigma F_{Y}=0\right)$ (class)

\section{Beyond Activities:}

These activities should be designed to provide students the opportunity to practice their new knowledge of writing force vectors in component form and using that form within an equilibrium design context.

Additionally, the discussions about these activities should explicitly highlight that the use of vectors, especially in component form, to represent forces is used primarily in engineering for the ease this mathematical form allows in the addition of forces to satisfy equilibrium requirements.

Paper formulation, using FBD and component force vector expression, for a more complex 2-D rigid body equilibrium design problem (group project)

Class discussion of each group's paper formulation with emphasis on FBD and force vector representation.

Paper design solution, using FBD, component force vector representation, and mathematical expression for force equilibrium (group project/group discussion)

Physical fabrication of design solution (group)

Class discussion of each group's physical solution

Class discussion of ease of using component force vector form to add forces so as to solve equilibrium design problems.

This example of the iterative use of the Into, Through, and Beyond method to 'scaffold' student learning, from developing a physical understanding of the physical nature of forces and moments to mathematically expressing forces in component vector form to facilitate the force addition requirements of equilibrium, indicates how this method could be used in the teaching of Statics. Of course, the content sequencing and content activities used above serve only as an example. The use of different content sequencing, such as that proposed by Dollár and Steif, ${ }^{8}$ or the inclusion of a variety of hands-on activities or design problems, is certainly be possible within the flexible framework of the Into, Through, and Beyond methodology. Clearly the content sequencing and the activities used within this framework will require testing to ensure complete student comprehension and mastery of the requisite skills and engineering concepts of Statics through the use of this methodology. 


\section{Conclusion}

The Into, Through, and Beyond content-based instruction methodology has been used effectively in the teaching of reading, ESL, and foreign languages. The 'scaffolding' concept that essentially underlies this methodology provides a more focused, rigorous, and pedagogically-proven basis for the sequencing of materials than a more looselystructured and anecdotally-based approach of a) review/introduction, b) presentation of new materials, and c) application. Thus, this methodology provides a framework by which to effectively integrate many pieces of the Statics teaching puzzle by utilizing the pedagogically-proven 'scaffolding' approach.

In addition to providing a rigorous 'scaffolded' framework by which to teach Statics, this methodology highlights, through its tested linguistic usage, a crucial distinction between 'skills' and 'concept content' that should, through analogy, be made in the teaching of Statics skills (vocabulary and grammar/rules) and concepts. As stated earlier, it has been shown that student learning and retention of linguistic skills, i.e., the effective use of vocabulary and grammar, was improved when these linguistic basics were taught within a content-based framework. Similarly, student learning and retention of the content materials also has been shown to be improved through use of this teaching method. These results then indicate that it is important for us to make a clear distinction between Statics 'skills' and 'concept content' so that we can ensure that we truly are teaching 'skills' within a 'concept content' framework. However, the distinctions between 'skills' and 'concepts' is not as clear-cut in Statics, as it is in linguistics. For example, is a 'force' a 'vocabulary' that has a certain set of 'grammar' rules associated with it? If so, then might we more effectively teach those 'skills' within the 'concept content' of equilibrium, rather than outside of that context. However, if we define 'force' as a 'concept', what 'vocabulary' and 'grammar' skills should we teach within that specific 'concept content' framework? While these questions may on the surface seem semantic in nature, research that supports the effectiveness of teaching 'skills' within a 'concept content' framework suggests that these are questions that may well be worth our posing and answering.

\section{Acknowledgements}

The author gratefully acknowledges the support provided by the Mechanical Engineering Department at the Virginia Military Institute, especially the assistance generously provided by Captain Michael Sexton.

\section{Bibliographic Information}

1. Brinton, D.M. and Holten, C., "Into, Through, and Beyond: A Framework to Develop Content-Based Material", Forum, Vol. 35, No. 4, Oct.-Dec. 1997. 
2. Pally, M., "Sustaining Interest/Advancing Learning: Sustained Content-based Instruction in ESL/EFL Theoretical Background and Rationale", Chapter 1, Sustained Content Teaching in Academic ESL/EFL: A Practical Approach, M. Pally (ed.), Houghton-Mifflin, Boston, 2000.

3. Brinton, D.M. and Holten, C., p. 11.

4. Brinton, D.M. and Holten, C., p. 11.

5. Brinton, D.M. and Holten, C., p. 11.

6. Brinton, D.M. and Holten, C., p. 11.

7. Brinton, D.M. and Holten, C., p. 11.

8. Dollár, A. and Steif, P.S., "Reinventing the Teaching of Statics", Proceedings of the 2004 American Society for Engineering Education Annual Conference \& Exposition, Salt Lake, 2004.

\section{Biographical Information}

Associate Professor, Department of Mechanical Engineering, Virginia Military Institute, Lexington, VA. Degrees: B.S. M.E. 1982, University of South Carolina; M.S. M.E. 1991, University of South Carolina; Ph.D. Theor. and Appl. Mechanics 1998, University of Illinois at Urbana-Champaign.

Research areas: solid mechanics, materials, and engineering education. 